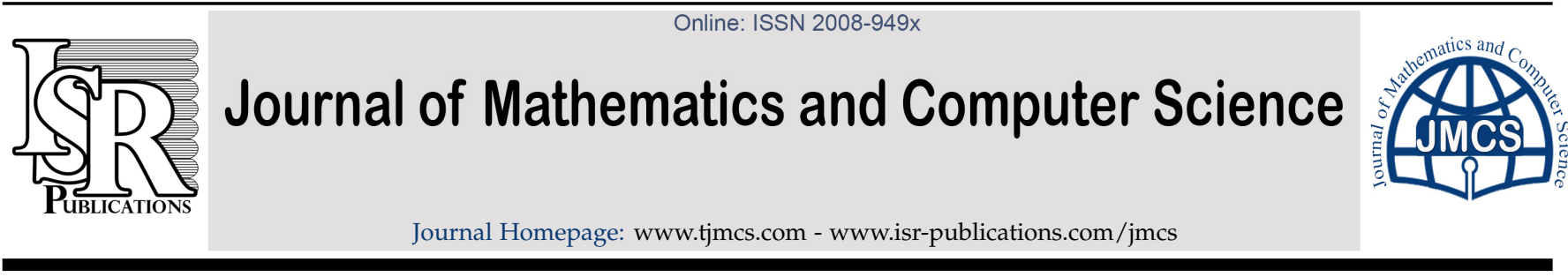

\title{
R-robustly measure expansive homoclinic classes are hyperbolic
}

\author{
Manseob Lee \\ Department of Mathematics, Mokwon University, Daejeon, 302-729, Korea.
}

\begin{abstract}
Let $f: M \rightarrow M$ be a diffeomorphism on a closed smooth $n(n \geqslant 2)$-dimensional manifold $M$ and let $p$ be a hyperbolic periodic point of $f$. We show that if the homoclinic class $H_{f}(p)$ is R-robustly measure expansive then it is hyperbolic.
\end{abstract}

Keywords: Expansive, measure expansive, local product structure, shadowing, hyperbolic, homoclinic class, generic. 2010 MSC: 34D10, 37C20, 37C29, 37C50, 37D30.

(c)2018 All rights reserved.

\section{Introduction}

Roughly speaking, definition of expansiveness is, if two orbits are closed then they are one orbit which was introduced by Utz [22]. A main research is to study structure of the orbits in differentiable dynamical systems, and so a goal of differentiable dynamical system is to study stability properties (Anosov, Axiom A, hyperbolic, structurally stable, etc.). Therefore, expansiveness is an important notion to study stability properties. For instance, Mañe [11] proved that if a diffeomorphism is $C^{1}$ robustly expansive then it is quasi-Anosov. Arbieto [1] proved that for $\mathrm{C}^{1}$ generic an expansive diffeomorphism is Axiom A without cycles. For expansivity, we can find various generalization notations, that is, continuum-wise expansive [5], n-expansive [13], and measure expansive [14]. Among that, we study measure expansiveness in the paper. Let $M$ be a closed smooth $n(n \geqslant 2)$-dimensional Riemmanian manifold, and let Diff $(M)$ be the space of diffeomorphisms of $M$ endowed with the $C^{1}$-topology. Denote by $d$ the distance on $M$ induced from a Riemannian metric $\|\cdot\|$ on the tangent bundle TM. Let $\Lambda$ be a closed f-invariant set. We say that $\Lambda$ is hyperbolic if the tangent bundle $T_{\Lambda} M$ has a Df-invariant splitting $E^{s} \oplus E^{\mathfrak{u}}$ and there exist constants $C>0$ and $0<\lambda<1$ such that

$$
\left\|\left.D_{\chi} f^{n}\right|_{E_{\chi}^{s}}\right\| \leqslant C \lambda^{n} \text { and }\left\|\left.D_{\chi} f^{-n}\right|_{E_{\chi}^{u}}\right\| \leqslant C \lambda^{n}
$$

for all $x \in \Lambda$ and $n \geqslant 0$. If $\Lambda=M$, then we say that $f$ is Anosov.

For any closed $f$-invariant set $\Lambda \subset M$, we say that $\Lambda$ is expansive for $f$, if there is $e>0$ such that for any $x, y \in \Lambda$ if $d\left(f^{n}(x), f^{n}(y)\right) \leqslant e$ then $x=y$. Equivalently, $\Lambda$ is expansive for $f$ if there is $e>0$ such that 
$\Gamma_{e}^{f}(x)=\{x\}$ for all $x \in \Lambda$, where $\Gamma_{e}^{f}(x)=\left\{y \in \Lambda: d\left(f^{i}(x), f^{i}(y)\right) \leqslant e\right.$ for all $\left.i \in \mathbb{Z}\right\}$. Let $\mathcal{M}(M)$ be the set of all Borel probability measures on $M$ endowed with the weak* topology, and let $\mathcal{M}^{*}(M)$ be the set of nonatomic measures $\mu \in \mathcal{M}(M)$. For any $\mu \in \mathcal{M}^{*}(M)$, we say that $\Lambda$ is $\mu$-expansive for $f$ if $\mu\left(\Gamma_{e}^{f}(x)\right)=0 . \Lambda$ is said to be measure expansive for $f$ if $\Lambda$ is $\mu$-expansive for all $\mu \in \mathcal{M}^{*}(M)$; that is, there is a constant $e>0$ such that for any $\mu \in \mathcal{M}^{*}(M)$ and $x \in \Lambda, \mu\left(\Gamma_{e}^{f}(x)\right)=0$. Here $e$ is called a measure expansive constant of $\left.f\right|_{\Lambda}$. Clearly, the expansiveness implies the measure expansiveness, but the converse does not hold in general (see [14, Theorem 1.35]). We say that $\mathrm{f}$ is quasi-Anosov if for any $v \in \mathrm{TM} \backslash\{0\}$, the set $\left\{\left\|\mathrm{D} f^{\mathrm{n}}(v)\right\|: \mathrm{n} \in \mathbb{Z}\right\}$ is unbounded. Sakai et al. [19] proved that if a diffeomorphism $f$ is $C^{1}$ robustly measure expansive then it is quasi-Anosov. Lee [7] proved that for $C^{1}$ generic $f$, if $f$ is measure expansive then it is Axiom A without cycles. It is well known that if $p$ is a hyperbolic periodic point of $f$ with period $\pi(p)$ then the sets

$$
W^{s}(p)=\left\{x \in M: f^{\pi(p) n}(x) \rightarrow p \text { as } n \rightarrow \infty\right\}
$$

and

$$
W^{\mathfrak{u}}(p)=\left\{x \in M: f^{-\pi(p) n}(x) \rightarrow p \text { as } n \rightarrow \infty\right\}
$$

are $C^{1}$-injectively immersed submanifolds of $M$. A point $x \in W^{s}(p) \pitchfork W^{\mathfrak{u}}(p)$ is called a homoclinic point of $f$ associated to $p$. The closure of the homoclinic points of $f$ associated to $p$ is called the homoclinic class of $f$ associated to $p$, and it is denoted by $H_{f}(p)$. It is clear that $H_{f}(p)$ is compact, transitive, and invariant.

Denote by $P(f)$ the set of all periodic points of $f$. Let $q$ be a hyperbolic periodic point of $f$. We say that $p$ and $q$ are homoclinically related, and write $p \sim q$ if

$$
W^{s}(p) \pitchfork W^{\mathfrak{u}}(q) \neq \emptyset \text { and } W^{\mathfrak{u}}(p) \pitchfork W^{s}(q) \neq \emptyset .
$$

It is clear that if $p \sim q$ then index $(p)=\operatorname{index}(q)$, that is, $\operatorname{dim} W^{s}(p)=\operatorname{dim}^{s}(q)$. By the Smale's transverse homoclinic point theorem, $H_{f}(p)=\overline{\left\{q \in P_{h}(f): q \sim p\right\}}$, where $\bar{A}$ is the closure of the set $A$ and $P_{h}(f)$ is the set of all hyperbolic periodic points. Note that if $p$ is a hyperbolic periodic point of $f$ then there is a neighborhood $U$ of $p$ and a $C^{1}$-neighborhood $U(f)$ of $f$ such that for any $g \in U(f)$ there exists a unique hyperbolic periodic point $p_{g}$ of $g$ in $U$ with the same period as $p$ and index $\left(p_{g}\right)=\operatorname{index}(p)$. Such a point $p_{g}$ is called the continuation of $p=p_{f}$. We say that $\Lambda$ is locally maximal if there is a neighborhood $\mathrm{U}$ of $\Lambda$ such that $\Lambda=\bigcap_{n \in \mathbb{Z}} \mathrm{f}^{\mathrm{n}}(\mathrm{U})$.

In differentiable dynamical systems, a main research topic is to study that for a given system, if the system has a property then we consider that a system which is $\mathrm{C}^{1}$-nearby system has the same property. Then, we consider various type of $\mathrm{C}^{1}$-perturbation property on a closed invariant set which are the following.

(a) We say that $\mathrm{H}_{\mathrm{f}}(\mathrm{p})$ is $\mathrm{C}^{1}$ robustly $\mathfrak{P}$ property if there is a $\mathrm{C}^{1}$-neighborhood $\mathcal{U}(\mathrm{f})$ of $f$ such that for any $g \in \mathcal{U}(f), H_{g}\left(p_{g}\right)$ is $\mathfrak{P}$ property. If $\mathfrak{P}$ is expansive then the expansive constant is uniform, which means that the constant only depends on $f$ (see $[15,16])$.

(b) We say that $\mathrm{H}_{\mathrm{f}}(\mathrm{p})$ is $\mathrm{C}^{1}$ persistently $\mathfrak{P}$ property if there is a $\mathrm{C}^{1}$-neighborhood $U(f)$ of $f$ such that for any $g \in \mathcal{U}(f), H_{g}\left(p_{g}\right)$ is $\mathfrak{P}$ property. If $\mathfrak{P}$ is expansive then the expansive constant is not uniform which means that the constant depends on $g \in \mathcal{U}(f)$ (see [20]).

(c) We say that $H_{f}(p)$ is $C^{1}$ stably $\mathfrak{P}$ property if there are a $C^{1}$-neighborhood $U(f)$ of $f$ and a neighborhood $U$ of $H_{f}(p)$ such that for any $g \in U(f), \Lambda_{g}(U)$ is $\mathfrak{P}$ property, where $\Lambda_{g}(U)=\bigcap_{n \in \mathbb{Z}} g^{n}(U)$ is the continuation of $\mathrm{H}_{\mathrm{f}}(\mathrm{p})$. If $\mathfrak{P}$ is expansive, then the expansive constant is not uniform which means that the constant depends on $g \in \mathcal{U}(f)$ (see [8]).

In the item (c), we can also consider a closed invariant set. We say that a subset $\mathcal{G} \subset \operatorname{Diff}(M)$ is residual if $\mathcal{G}$ contains the intersection of a countable family of open and dense subsets of $\operatorname{Diff}(M)$; in this case $\mathcal{G}$ is dense in $\operatorname{Diff}(M)$. A property $\mathcal{P}$ is said to be $\left(C^{1}\right)$-generic if $\mathcal{P}$ holds for all diffeomorphisms which belong to some residual subset of $\operatorname{Diff}(M)$.

$\mathrm{Li}$ [10] introduced another $\mathrm{C}^{1}$ robust property which is called $R$-robustly $\mathfrak{P}$ property. Using to the notion, we consider the following. 
Definition 1.1. Let the homoclinic class $H_{f}(p)$ associated to a hyperbolic periodic point $p$. We say that $\mathrm{H}_{\mathrm{f}}(\mathrm{p})$ is $R$-robustly measure expansive if there are a $C^{1}$-neighborhood $\mathcal{U}(\mathrm{f})$ of $f$ and a residual set $\mathcal{G}$ of $\mathcal{U}(\mathrm{f})$ such that for any $g \in \mathcal{G}, \mathrm{H}_{g}\left(p_{g}\right)$ is measure expansive, where $p_{g}$ is the continuation of $p$.

Recently, Pacificao and Vieites [17] proved that a diffeomorphism $f$ in a residual subset far from homoclinic tangencies are measure expansive. Lee and Lee [9] proved that if the homoclinic class $H_{f}(p)$ is $C^{1}$ stably measure expansive then it is hyperbolic. Koo et al. [6] proved that for $C^{1}$ generic $f$, if a locally maximal homoclinic class $\mathrm{H}_{\mathrm{f}}(\mathrm{p})$ is measure expansive, then it is hyperbolic. Owing to the result, we have the following which is a main theorem of the paper.

Theorem 1.2. Let the homoclinic class $\mathrm{H}_{\mathrm{f}}(\mathrm{p})$ associated to a hyperbolic periodic point $\mathrm{p}$. If $\mathrm{H}_{\mathrm{f}}(\mathrm{p})$ is $R$-robustly measure expansive then it is hyperbolic.

\section{Dominated splitting and Hyperbolic periodic points in $H_{f}(p)$}

Let $M$ be as before, and let $f \in \operatorname{Diff}(M)$. A periodic point for $f$ is a point $p \in M$ such that $f^{\pi(p)}(p)=p$, where $\pi(p)$ is the minimum period of $p$. Denote by $P(f)$ the set of all periodic points of $f$. For given $x, y \in M$, we write $x \rightarrow y$ if for any $\delta>0$, there is a $\delta$-pseudo orbit $\left\{x_{i}\right\}_{i=0}^{n}(n>1)$ of $f$ such that $x_{0}=x$ and $x_{n}=y$. We write $x \leftrightarrow y$ if $x \rightarrow y$ and $y \rightarrow x$. The set of points $\{x \in M: x \leftrightarrow x\}$ is called the chain recurrent set of $f$ and is denoted by $\mathcal{R}(f)$. It is clear that $P(f) \subset \Omega(f) \subset \mathcal{R}(f)$. Here $\Omega(f)$ is the non-wandering set of $f$. Let $p$ be a hyperbolic periodic point of $f$. We say that the chain component if for any $x \in M, x \rightarrow p$ and $p \rightarrow x$ and denote it by $C_{f}(p)$. Note that the chain component $C_{f}(p)$ of $f$ is a equivalent class, it is a closed set and f-invariant set. The following was proved by Bonatti and Crovisier [2].

Remark 2.1. There is a residual set $\mathcal{G}_{1} \subset \operatorname{Diff}(M)$ such that for any $f \in \mathcal{G}_{1}, H_{g}(p)=C_{f}(p)$ for some hyperbolic periodic point $p$.

Proposition 2.2. Let the homoclinic class $\mathrm{H}_{\mathrm{f}}(\mathrm{p})$ be R-robustly measure expansive. If $\mathrm{x} \in \mathrm{W}^{\mathrm{s}}(\mathrm{p}) \cap \mathrm{W}^{\mathrm{u}}(\mathrm{p})$, then $x \in W^{s}(p) \pitchfork W^{u}(p)$.

Proof. Since $\mathrm{H}_{\mathrm{f}}(\mathrm{p})$ is R-robustly measure expansive, there exists a $C^{1}$-neighborhood $U(f)$ and a residual set $\mathcal{G} \subset \mathcal{U}(f)$ such that for any $g \in \mathcal{G}, H_{g}\left(p_{g}\right)$ is measure expansive. Let $\mathcal{G}=\mathcal{G}_{1}$. Since $x \in W^{s}(p) \cap W^{\mathfrak{u}}(p)$, by [17, Proposition 2.6], there is $g \in \mathcal{U}(f) \cap \mathcal{G}$ such that we can make a small arc $\mathcal{J} \subset W^{s}\left(p_{g}\right) \cap W^{u}\left(p_{g}\right)$. Since $H_{g}\left(p_{g}\right)=C_{g}\left(p_{g}\right)$, we know $\mathcal{J} \subset C_{g}\left(p_{g}\right)$. Let $\operatorname{diam}(\mathcal{J})=l$. We define a measure $\mu \in \mathcal{M}^{*}(M)$ by $\mu(C)=v(C \cap \mathcal{J})$ for any Borel set $C$ of $M$, where $v$ is a normalized Lebesgue measure on $\mathcal{J}$. Let $e=l / 4$ be a measure expansive constant. Since $\mathcal{J} \subset W^{s}\left(p_{g}\right) \cap W^{\mathfrak{u}}\left(p_{g}\right)$, there is $N>0$ such that $\operatorname{diam}\left(g^{i}(\mathcal{J})\right) \leqslant e / 4$ for $-N \leqslant i \leqslant N$, and $g^{i}(\mathcal{J}) \subset W_{e / 4}^{s}\left(p_{g}\right) \cap W_{e / 4}^{u}\left(p_{g}\right)$ for $|i|>N$. Thus for all $i \in \mathbb{Z}$, we know that $\operatorname{diam}\left(g^{i}(\mathcal{J})\right) \leqslant e$. Recall that

$$
\Gamma_{e}(x)=\left\{y \in H_{g}\left(p_{g}\right): d\left(g^{i}(x), g^{i}(y)\right) \leqslant e \text { for } i \in \mathbb{Z}\right\} .
$$

We can construct the set

$$
A_{e}(x)=\left\{y \in \mathcal{J}: d\left(g^{i}(x), g^{i}(y)\right) \leqslant e \text { for } i \in \mathbb{Z}\right\} .
$$

Then we know $A_{e}(x) \subset \Gamma_{e}(x)$. Thus we have

$$
0<\mu\left(A_{e}(x)\right) \leqslant \mu\left(\Gamma_{e}(x)\right)
$$

which is a contradiction to the measure expansivity of $\mathrm{H}_{g}\left(\mathrm{p}_{g}\right)$.

For $f \in \operatorname{Diff}(M)$, we say that a compact $f$-invariant set $\Lambda$ admits a dominated splitting if the tangent bundle $T_{\wedge} M$ has a continuous Df-invariant splitting $E \oplus F$ and there exist $C>0,0<\lambda<1$ such that for all $x \in \Lambda$ and $n \geqslant 0$, we have

$$
\left\|\left.D f^{n}\right|_{E(x)}\right\| \cdot\left\|\left.D f^{-n}\right|_{F\left(f^{n}(x)\right)}\right\| \leqslant C \lambda^{n} .
$$


Theorem 2.3. Let $\mathrm{H}_{\mathrm{f}}(\mathrm{p})$ be the homoclinic class containing a hyperbolic periodic point $\mathrm{p}$. Suppose that $\mathrm{H}_{\mathrm{f}}(\mathrm{p})$ is $R$-robustly measure expansive. Then there exist a $C^{1}$-neighborhood $U(f)$ of $f$ and a residual set $\mathcal{G} \subset U(f)$ such that for any $\mathrm{g} \in \mathcal{G}, \mathrm{H}_{\mathrm{g}}\left(\mathrm{p}_{\mathrm{g}}\right)$ admits a dominated splitting $\mathrm{T}_{\mathrm{H}_{\mathrm{g}}\left(\mathrm{p}_{\mathrm{g}}\right)} \mathrm{M}=\mathrm{E}(\mathrm{g}) \oplus \mathrm{F}(\mathrm{g})$ with index $\left(\mathrm{p}_{\mathrm{g}}\right)=\operatorname{dimE}(\mathrm{g})$.

Proof. Suppose that $\mathrm{H}_{\mathrm{f}}(\mathrm{p})$ is R-robustly measure expansive. Then as in the proof of [20, Theorem 1], there is $m>0$ such that for every $x \in W^{s}(p) \pitchfork W^{u}(p)$ there exists $m_{1} \in[90, m]$ such that $\left\|\left.D f^{m_{1}}\right|_{E(x)}\right\|$. $\left\|\left.D f^{-m_{1}}\right|_{F\left(f^{m_{1}}(x)\right)}\right\| \leqslant 1 / 2$. Since the dominated splitting can be extended by continuity to the

$$
\overline{W^{s}(p) \pitchfork W^{\mathfrak{u}}(p)}=H_{f}(p),
$$

we have that $H_{f}(p)$ has a dominated splitting $E \oplus F$.

Theorem 2.4. Let the homoclinic class $\mathrm{H}_{\mathrm{f}}(\mathrm{p})$ be R-robustly measure expansive. Then there exist $\mathrm{C}>0,0<\lambda<1$ and $\mathrm{m}>0$ such that $\mathrm{q}$ is a hyperbolic periodic point of period $\pi(\mathrm{q})$ and $\mathrm{q} \sim \mathrm{p}$, then

$$
\prod_{i=0}^{k-1}\left\|\left.D f^{m}\right|_{E^{s}\left(f^{i m}(q)\right)}\right\|<C \lambda^{k} \text { and } \prod_{i=0}^{k-1}\left\|\left.D f^{-m}\right|_{E^{u}\left(f^{-i m}(q)\right)}\right\|<C \lambda^{k},
$$

where $\mathrm{k}=[\pi(\mathrm{q}) / \mathrm{m}]([\cdot]$ represents the integer part).

Proof. Since $H_{f}(p)$ is R-robustly measure expansive, there are a $C^{1}$-neighborhood $U(f)$ and a residual set $\mathcal{G} \subset \mathcal{U}(f)$ such that for any $g \in \mathcal{G}, H_{g}\left(p_{g}\right)$ is measure expansive. Let $\mathcal{G}=\mathcal{G}_{1}$. Since $q \in H_{f}(p)$ and $p \sim q$, as in the proof of [20], it is enough to show that the family of periodic sequences of linear isomorphisms of $\mathbb{R}^{\mathfrak{n}}$ generated by Df along the hyperbolic periodic points $q \in H_{f}(p), q \sim p$ and index $(p)=\operatorname{index}(q)$ is uniformly hyperbolic. Suppose, by contradiction, that the assume does not hold. Then as in the proof of [18, Theorem B], we may assume that a hyperbolic periodic point $q \in H_{f}(p)$ such that the weakest normalized eigenvalue $\lambda$ is close to 1 . Then by Franks lemma, there is $g \in \mathcal{G}$ such that for any small $\gamma>0$ we can construct a closed small curve $\mathcal{J}_{q}$ containing $q$ or a closed small circle $\mathcal{C}_{\mathrm{q}}$ centered at $q$ such that $\mathcal{J}_{q} \subset \mathrm{C}_{g}\left(p_{g}\right)$ and two endpoints are related to $p_{g}$ and $\mathcal{C}_{q} \subset \mathrm{C}_{g}\left(p_{g}\right)$. Note that $\mathcal{J}_{q}$ and $\mathcal{C}_{q}$ are $g^{\pi(q)}$-invariant, normally hyperbolic, and $\left.g^{l \pi(q)}\right|_{\mathcal{J}_{q}}$ is the identity map for some $l>0$ (see [18]). For $\mathcal{J}_{q}$, we define a measure $\mu \in \mathcal{M}^{*}(M)$ by

$$
\mu(C)=\frac{1}{l \pi(q)} \sum_{i=0}^{l \pi(q)-1} v\left(g^{-i}\left(C \cap g^{i}\left(\mathcal{J}_{q}\right)\right)\right)
$$

for any Borel set $C$ of $M$, where $v$ is a normalized Lebesgue measure on $\mathcal{J}_{q}$. Let $\gamma \leqslant e$ be a measure expansive constant of $\left.g\right|_{H_{g}\left(p_{g}\right)}$. By [14, Proposition], $g$ is measure expansive if and only if $g^{n}$ is measure expansive for $n \in \mathbb{Z} \backslash\{0\}$. Let $\Gamma_{e}^{g}(x)=\left\{y \in H_{g}\left(p_{g}\right): d\left(g^{l \pi(q) i}(x), g^{l \pi(q) i}(y)\right) \leqslant e\right.$, for all $\left.i \in \mathbb{Z}\right\}$. Then we have

$$
\left\{y \in \mathcal{J}_{q}: d\left(g^{l \pi(q) i}(x), g^{l \pi(q) i}(y)\right) \leqslant e \text { for all } i \in \mathbb{Z}\right\}=\left\{y \in \mathcal{J}_{q}: d\left(g^{i}(x), g^{i}(y)\right) \leqslant e \text { for all } i \in \mathbb{Z}\right\} .
$$

Thus we know

$$
0<\mu\left(\left\{y \in \mathcal{J}_{q}: d\left(g^{i}(x), g^{i}(y)\right) \leqslant e \text { for all } i \in \mathbb{Z}\right\}\right) \leqslant \mu\left(\Gamma_{e}^{g}(x)\right) .
$$

Since $H_{g}\left(p_{g}\right)$ is measure expansive for $g$, we know $\mu\left(\Gamma_{e}^{g}(x)\right)=0$. Thus we have

$$
\mu\left(\left\{y \in \mathcal{J}_{q}: d\left(g^{i}(x), g^{i}(y)\right) \leqslant e \text { for all } i \in \mathbb{Z}\right\}\right)=0 .
$$

This is a contradiction.

For $\mathcal{C}_{\mathrm{q}}$ case, if $\mathcal{C}_{\mathrm{q}}$ is irrational rotation then using the Franks' lemma, there is $h \in \mathcal{U}(\mathrm{g}) \cap \mathcal{G}$ such that $\mathcal{C}_{q_{h}}$ is rational rotation which is centered at $q_{h}$, where $\mathcal{U}(\mathrm{g})$ is a $C^{1}$-neighborhood of $g$, and $q_{h}$ is the 
continuation of $q$ for $h$. Then there is $k>0$ such that $h^{k}: \mathcal{C}_{q_{h}} \rightarrow \mathcal{C}_{q_{h}}$ is the identity map. Then we define a measure $\mu \in \mathcal{M}^{*}(M)$ by

$$
\mu(B)=\frac{1}{k} \sum_{i=0}^{k-1} \eta\left(h^{i}\left(B \cap h^{-i}\left(\mathcal{C}_{q_{h}}\right)\right)\right.
$$

for any Borel set $B$ of $M$, where $\eta$ is a normalized Lebesgue measure on $\mathcal{C}_{q_{h}}$. Then as in the proof of previous argument, we can derive a contradiction.

By [14, Proposition], $g$ is measure expansive if and only if $\mathrm{g}^{\mathrm{n}}$ is measure expansive for $n \in \mathbb{Z} \backslash\{0\}$. Theorem 2.4 can be rewritten as the following.

Theorem 2.5. Let the homoclinic class $\mathrm{H}_{\mathrm{f}}(\mathrm{p})$ be R-robustly measure expansive. Then there exist $0<\lambda<1$ and $\mathrm{L} \leqslant 1$ such that $\mathrm{q}$ is a hyperbolic periodic point of period $\pi(\mathrm{q})$ with $\mathrm{L}>\pi(\mathrm{q})$ and $\mathrm{q} \sim \mathrm{p}$, then

$$
\prod_{i=0}^{\pi(q)-1}\left\|\left.D f\right|_{E^{s}\left(f^{i}(q)\right)}\right\|<\lambda^{\pi(q)} \text { and } \prod_{i=0}^{\pi(q)-1}\left\|\left.D f^{-1}\right|_{E^{u}\left(f^{-i}(q)\right)}\right\|<\lambda^{\pi(q)} .
$$

\section{Local product structure}

Let $\Lambda$ be a closed, f-invariant set. We say that $\Lambda$ has a local product structure if for given $\epsilon>0$ there exists a $\delta>0$ such that if $d(x, y)<\delta$ and $x, y \in \Lambda$, then

$$
\emptyset \neq W_{\epsilon}^{s}(x) \cap W_{\epsilon}^{u}(y) \subset \Lambda .
$$

By the uniqueness of the dominated splitting, if $q \in \mathrm{H}_{\mathrm{f}}(\mathrm{p})$ is a periodic point with $q \sim p$ then we have $E(q)=E^{s}(q)$ and $F(q)=E^{\mathfrak{u}}(q)$. Let $\operatorname{dim} E=s$ and by $\operatorname{dim} F=u$, and put $D_{r}^{j}=\left\{x \in \mathbb{R}^{j}:\|x\| \leqslant r\right\}(r>0)$, for $j=s, u$. Let $E m b_{\wedge}\left(D_{1}^{j}, M\right)$ be the space of $C^{1}$ embeddings $\beta: D_{1}^{j} \rightarrow M$ such that $\beta(0) \in \Lambda$ endowed with the $C^{1}$ topology. Then we have the following.

Proposition 3.1 ( $[4,12])$. Let $\mathrm{H}_{\mathrm{f}}(\mathrm{p})$ be the homoclinic class of $\mathrm{f}$ associated to a hyperbolic periodic point $\mathrm{p}$, and let $\Lambda=\mathrm{H}_{\mathrm{f}}(\mathrm{p})$. Suppose that $\Lambda$ has a dominated splitting $\mathrm{E} \oplus \mathrm{F}$. Then there exist sections $\phi^{\mathrm{s}}: \Lambda \rightarrow \operatorname{Emb}_{\wedge}\left(\mathrm{D}_{1}^{\mathrm{s}}, \mathrm{M}\right)$ and $\phi^{\mathrm{u}}: \Lambda \rightarrow \mathrm{Emb}_{\Lambda}\left(\mathrm{D}_{1}^{\mathrm{u}}, \mathrm{M}\right)$ such that by defining $\mathrm{W}_{\epsilon}^{\mathrm{cs}}(\mathrm{x})=\phi^{\mathrm{s}}(\mathrm{x}) \mathrm{D}_{\epsilon}^{\mathrm{s}}$ and $\mathrm{W}_{\epsilon}^{\mathrm{cu}}(\mathrm{x})=\phi^{\mathrm{u}}(\mathrm{x}) \mathrm{D}_{\epsilon}^{\mathrm{u}}$, for each $x \in \Lambda$, we have

(1) $\mathrm{T}_{\mathrm{x}} \mathrm{W}_{\epsilon}^{\mathrm{cs}}(\mathrm{x})=\mathrm{E}(\mathrm{x})$ and $\mathrm{T}_{\mathrm{x}} \mathrm{W}_{\epsilon}^{\mathrm{cu}}(\mathrm{x})=\mathrm{F}(\mathrm{x})$;

(2) for every $0<\epsilon_{1}<1$ there exists $0<\epsilon_{2}<1$ such that $f\left(W_{\epsilon_{2}}^{c s}(x)\right) \subset W_{\epsilon_{1}}^{c s}(f(x))$ and $f^{-1}\left(W_{\epsilon_{2}}^{c u}(x)\right) \subset$ $W_{\epsilon_{1}}^{c s}\left(f^{-1}(x)\right)$

(3) for every $0<\epsilon_{1}<1$ there exists $0<\delta<1$ such that if $\mathrm{d}(x, y)<\delta(x, y \in \Lambda)$ then $W_{\epsilon_{1}}^{c s}(x) \cap W_{\epsilon_{1}}^{c u}(y) \neq \emptyset$, and this intersection is transverse.

The sets $W_{\epsilon}^{c s}(x)$ and $W_{\epsilon}^{c u}(x)$ are called the local center stable and local unstable manifolds of $x$, respectively. The following lemma can be proved similarly to that of Lemma 4 in [20].

Lemma 3.2. Let $\mathrm{H}_{\mathrm{f}}(\mathrm{p})$ be the homoclinic class of $\mathrm{f}$ associated to a hyperbolic periodic point $\mathrm{p}$, and suppose that $\mathrm{H}_{\mathrm{f}}(\mathrm{p})$ is $R$-robustly measure expansive. Then for $\mathrm{C}, \lambda$ as in Theorem 3.1 and $\delta>0$ satisfying $\lambda^{\prime}=\lambda(1+\delta)<1$ and $q \sim p$, there exists $0<\epsilon_{1}<\epsilon$ such that if for all $0 \leqslant n \leqslant \pi(q)$ it holds that for some $\epsilon_{2}>0, f^{n}\left(W_{\epsilon_{2}}^{c s}(q)\right) \subset$ $W_{\epsilon_{1}}^{c s}\left(f^{n}(q)\right)$, then

$$
f^{\pi(q)}\left(W_{\epsilon_{2}}^{c s}(q)\right) \subset W_{C \lambda^{\prime \pi(q)} \epsilon_{2}}^{c s}(q) .
$$

Similarly, if $\mathrm{f}^{-\mathrm{n}}\left(\mathbf{W}_{\epsilon_{2}}^{\mathrm{cu}}(\mathbf{q})\right) \subset \mathbf{W}_{\epsilon_{1}}^{\mathrm{cu}}\left(\mathrm{f}^{-\mathrm{n}}(\mathbf{q})\right)$, then

$$
f^{-\pi(q)}\left(W_{\epsilon_{2}}^{c u}(q)\right) \subset W_{C \lambda^{\prime \pi(q)} \epsilon_{2}}^{c u}(q) .
$$


Recall that by using the Smale's transverse theorem, we have $\mathrm{H}_{\mathrm{f}}(\mathrm{p})=\overline{\text { homo }}_{\mathrm{p}}$, where homo $\mathrm{p}=$ $\left\{q \in P_{h}(f): q \sim p\right\}$.

Lemma 3.3. Let $\mathrm{H}_{\mathrm{f}}(\mathrm{p})$ be the homoclinic class of $\mathrm{f}$ associated to a hyperbolic periodic point $\mathrm{p}$, and let $\mathrm{e}>0$ be $a$ measure expansive constant. Suppose that $\mathrm{H}_{\mathrm{f}}(\mathrm{p})$ is R-robustly measure expansive. Then

(a) for any hyperbolic periodic point $\mathrm{q} \in$ homo $_{\mathrm{p}}$ and $0<\epsilon_{1}<\mathrm{e}$, there is $\epsilon_{2}>0$ such that

$$
f^{n}\left(W_{\epsilon_{2}}^{c s}(q)\right) \subset W_{\epsilon_{1}}^{c s}\left(f^{n}(q)\right) \text { and } f^{-n}\left(W_{\epsilon_{2}}^{c u}(q)\right) \subset W_{\epsilon_{1}}^{c u}\left(f^{-n}(q)\right) \text { for all } n \geqslant 0 .
$$

(b) for any $\mathrm{y} \in \mathrm{W}_{\epsilon_{2}}^{\mathrm{cs}}(\mathrm{q})$ and $\mathrm{q} \in$ homo $_{\mathrm{p}}$ we have

$$
\lim _{n \rightarrow \infty} d\left(f^{n}(q), f^{n}(y)\right)=0 .
$$

Proof. Let $f \in \mathcal{G}=\mathcal{G}_{1}$ and let $\mathrm{H}_{\mathrm{f}}(\mathrm{p})$ is R-robustly measure expansive. To prove (a), it is enough to show that $f^{n}\left(W_{\epsilon_{2}}^{c s}(q)\right) \subset W_{\epsilon_{1}}^{c s}\left(f^{n}(q)\right)$. Let $\sup \left\{\operatorname{dim}_{\epsilon_{1}}^{c s}(q): q \in\right.$ homo $\left._{p}\right\}<$ e. Since $q \in$ homo $o_{p}$, we define

$$
\epsilon(q)=\sup \left\{\epsilon>0: f^{n}\left(W_{\epsilon}^{c s}(q)\right) \subset W_{\epsilon_{1}}^{c s}\left(f^{n}(q)\right) \text { for all } n \geqslant 0\right\} .
$$

By Proposition 3.1 and Lemma 3.2, $\epsilon(q)>0$. Let $\epsilon_{0}=\inf \{\epsilon(q): q \in$ homo $\}$. If $\epsilon_{0}>0$ then it is a proof of (a). Suppose, by contradiction, that there is a sequence $\left\{q_{n}\right\} \subset$ homo $o_{p}$ such that $\epsilon\left(q_{n}\right) \rightarrow 0$ as $n \rightarrow \infty$. Then we have $0<m_{n}<\pi\left(q_{n}\right)$ and $y_{n} \in W_{\epsilon\left(q_{n}\right)}^{c s}\left(q_{n}\right)$ such that $d\left(f^{m_{n}}\left(q_{n}\right), f^{m_{n}}\left(y_{n}\right)\right)=\epsilon_{1}$ for $f^{m_{n}}\left(q_{n}\right), f^{m_{n}}\left(y_{n}\right) \in W_{\epsilon\left(q_{n}\right)}^{c s}\left(q_{n}\right)$. Let $I_{n}$ be a closed connected arc joining $f^{m_{n}}\left(q_{n}\right)$ with $f^{m_{n}}\left(y_{n}\right)$. Then we know that

(i) $\mathrm{I}_{\mathrm{n}} \subset \mathrm{W}_{\epsilon\left(\mathrm{q}_{n}\right)}^{\mathrm{cs}}\left(\mathrm{q}_{\mathrm{n}}\right)$;

(ii) $f^{i}\left(I_{n}\right) \subset W_{\epsilon_{1}}^{c s}\left(f^{i}\left(q_{n}\right)\right)$ for $0 \leqslant i \leqslant \pi\left(q_{n}\right)$;

(iii) $\operatorname{diam}\left(\mathrm{I}_{\mathrm{n}}\right)=\epsilon_{1}$.

By Lemma 3.2, we know $f^{\pi\left(q_{n}\right)}\left(W_{\epsilon\left(q_{n}\right)}^{c s}\left(q_{n}\right)\right) \subset W_{C \lambda^{\prime \pi\left(q_{n}\right)} \epsilon\left(q_{n}\right)}^{c s}\left(q_{n}\right)$. Observe that if $n \rightarrow \infty$ then $m_{n} \rightarrow$ $\infty$ and $\pi\left(q_{n}\right)-m_{n} \rightarrow \infty$. Suppose that $f^{m_{n}}\left(q_{n}\right) \rightarrow x$ and $f^{m_{n}}\left(y_{n}\right) \rightarrow y$ as $n \rightarrow \infty$. Then $I_{n} \rightarrow I$, where $I$ is a close connected arc joining $x$ with $y$. It means that $\operatorname{diam}\left(f^{j}(I)\right) \leqslant \epsilon_{1}$ for all $j \in \mathbb{Z}$, and $x \in \overline{h_{0 m o}}=H_{f}(p)$. We show that the closed connected arc $I \subset H_{f}(p)$. Since $f \in \mathcal{G}, H_{f}(p)=C_{f}(p)$. For any $a \in I$, take $a_{n} \in W_{\epsilon\left(q_{n}\right)}^{c s}\left(q_{n}\right)$ such that $f^{m_{n}}\left(a_{n}\right) \rightarrow a$ as $n \rightarrow \infty$. As in the proof of [21, Lemma 2.6], let $\epsilon>0$ be arbitrary. Let $n \in \mathbb{N}$ be such that $\epsilon\left(q_{n}\right)<\epsilon$. Then for $n$ sufficiently large, $\left\{q_{n}, f\left(a_{n}\right), \ldots, f^{m_{n}-1}\left(a_{n}\right), a, f^{m_{n}+1}\left(a_{n}\right), \ldots, f^{\pi\left(q_{n}\right)-1}\left(a_{n}\right), q_{n}\right\}$ is a periodic $\epsilon$-chain through a and having a point in $H_{f}(p)$. Since $q_{n} \in$ homo $_{p}, H_{f}\left(q_{n}\right)=H_{f}(p)=C_{f}\left(q_{n}\right)=C_{f}(p)$ and so the closed connected arc I $\subset H_{f}(p)$. We define a measure $\mu \in \mathcal{M}^{*}(M)$ by $\mu(C)=\mu_{I}(C \cap I)$ for any Borel set $C$ of $M$, where $\mu_{\mathrm{I}}$ is a normalized Lebesgue measure on I. Let

$$
\Gamma_{e}(x)=\left\{y \in H_{f}(p): d\left(f^{i}(x), f^{i}(y)\right) \leqslant e \text { for } i \in \mathbb{Z}\right\} .
$$

Since for all $i \in \mathbb{Z}, \operatorname{diam}\left(f^{i}(I)\right) \leqslant e$, we can construct the set

$$
\left\{y \in I: d\left(f^{i}(x), f^{i}(y)\right) \leqslant e \text { for } i \in \mathbb{Z}\right\} .
$$

Then we know $\left\{y \in I: d\left(f^{i}(x), f^{i}(y)\right) \leqslant e\right.$ for $\left.i \in \mathbb{Z}\right\} \subset \Gamma_{e}(x)$. Thus we have

$$
0<\mu\left(\left\{y \in I: d\left(f^{i}(x), f^{i}(y)\right) \leqslant e \text { for } i \in \mathbb{Z}\right\}\right) \leqslant \mu\left(\Gamma_{e}(x)\right) .
$$

Since $H_{f}(p)$ is measure expansive, $\mu\left(\Gamma_{e}(x)\right)=0$. Thus $\mu\left(\left\{y \in I: d\left(f^{i}(x), f^{i}(y)\right) \leqslant e\right.\right.$ for $\left.\left.i \in \mathbb{Z}\right\}\right)=0$ which is a contradiction.

The proof of (b) is similar as in the proof of item (b) of [21, Lemma 2.6]. 
Remark 3.4. In the Lemma 3.3, we consider $q \in$ homo $_{p}$. Then we can extend $x \in \mathrm{H}_{\mathrm{f}}(\mathrm{p})$, that is, for any $x \in H_{f}(p)$ and $\epsilon_{1}>0$ there exists $\epsilon_{2}>0$ such that $f^{n}\left(W_{\epsilon_{2}}^{c s}(x)\right) \subset W_{\epsilon_{1}}^{c s}\left(f^{n}(x)\right)$ for all $n \geqslant 0$. And if $z \in W_{\epsilon_{2}}^{c s}(x)$ and $z \in H_{f}(p)$, then $d\left(f^{i}(z), f^{i}(x)\right) \rightarrow 0$ as $n \rightarrow \infty$.

Proposition 3.5. Suppose that the homoclinic class $\mathrm{H}_{\mathrm{f}}(\mathrm{p})$ is R-robustly measure expansive. Then $\mathrm{H}_{\mathrm{f}}(\mathrm{p})$ has a local product structure.

Proof. By Lemma 3.3, there is $\epsilon_{2}>0$ such that for any $q \in$ homo

$$
W_{\epsilon_{2}}^{c s}(q)=W_{\epsilon_{2}}^{s}(q) \text { and } W_{\epsilon_{2}}^{c u}(q)=W_{\epsilon_{2}}^{u}(q) .
$$

By Proposition 3.1 (3), there is $\delta>0$ such that for any $q, r \in$ homo $_{p}$,

$$
W_{\epsilon_{2}}^{s}(q) \cap W_{\epsilon_{2}}^{u}(r) \neq \emptyset \text {. }
$$

By $\lambda$-lemma, $W_{\epsilon_{2}}^{s}(q) \subset \overline{W^{s}(p)}$ and $W_{\epsilon_{2}}^{u}(r) \subset \overline{W^{u}(p)}$. Thus we know that $W_{\epsilon_{2}}^{s}(q) \cap W_{\epsilon_{2}}^{u}(r) \subset H_{f}(p)$. This means that $H_{f}(p)$ has a local product structure.

Corollary 3.6. Suppose that the homoclinic class $\mathrm{H}_{\mathrm{f}}(\mathrm{p})$ is R-robustly measure expansive. Then for any hyperbolic periodic point $\mathrm{q} \in \mathrm{H}_{\mathrm{f}}(\mathrm{p})$, index $(\mathrm{p})=\operatorname{index}(\mathrm{q})$.

Proof. The proof is directly obtained by Proposition 3.1 (3), Lemma 3.3, and Proposition 3.5. Thus for any hyperbolic periodic point $\mathrm{q} \in \mathrm{H}_{\mathrm{f}}(\mathrm{p})$,

$$
W^{s}(p) \pitchfork W^{\mathfrak{u}}(q) \neq \emptyset \text { and } W^{\mathfrak{u}}(p) \pitchfork W^{s}(q) \neq \emptyset .
$$

Thus we have $i n d e x(p)=\operatorname{index}(q)$.

\section{Proof of Theorem 1.2}

For any $\delta>0$, a sequence $\left\{x_{i}\right\}_{i \in \mathbb{Z}}$ is a $\delta$-pseudo orbit of $f$ if $d\left(f\left(x_{i}\right), x_{i+1}\right)<\delta$ for all $i \in \mathbb{Z}$. Let $\Lambda$ be a closed $f$-invariant set. We say that $f$ has the shadowing property on $\Lambda$ such that for any $\epsilon>0$ there is $\delta>0$ such that for any $\delta$-pseudo orbit $\left\{x_{i}\right\}_{i \in \mathbb{Z}} \subset \Lambda$ there is $z \in M$ such that $d\left(f^{i}(z), x_{i}\right)<\in$ for all $i \in \mathbb{Z}$. The following proposition is a very useful result for proving of Theorem 1.2.

Proposition 4.1 ([23, Proposition 3.3]). Let $\mathrm{p}$ be a hyperbolic periodic point, and let $\mathrm{H}_{\mathrm{f}}(\mathrm{p})$ be the homoclinic class of $\mathrm{f}$ containing $\mathrm{p}$. Let $0<\lambda<1$ and $\mathrm{L} \geqslant 1$ be given. Assume that $\mathrm{H}_{\mathrm{f}}(\mathrm{p})$ satisfies the following properties.

(1) There is a continuous Df-invariant splitting $\mathrm{T}_{\mathrm{H}_{\mathrm{f}}(\mathrm{p})} \mathrm{M}=\mathrm{E} \oplus \mathrm{F}$ with $\operatorname{dimE}=\operatorname{index}(\mathrm{p})$ such that for any $x \in H_{f}(p)$,

$$
\left\|\left.\mathrm{Df}\right|_{\mathrm{E}(\mathrm{x})}\right\| / \mathrm{m}\left(\left.\mathrm{Df}\right|_{\mathrm{F}(\mathrm{x})}\right)<\lambda^{2},
$$

where $\mathrm{m}(\mathrm{A})=\inf \|\| \mathrm{A}\|:\| v \|=1\}$ denotes the mininorm of a linear map $A$.

(2) For any $\mathrm{q} \in \mathrm{H}_{\mathrm{f}}(\mathrm{p}) \cap \mathrm{P}(\mathrm{f})$, if $\mathrm{q}$ is hyperbolic and $\pi(\mathrm{q})>\mathrm{L}$, then $\operatorname{index}(\mathrm{p})=\operatorname{index}(\mathrm{q})$ and

$$
\prod_{i=0}^{\pi(q)-1}\left\|\left.D f\right|_{E^{s}\left(f^{i}(q)\right)}\right\|<\lambda^{\pi(q)}, \quad \prod_{i=0}^{\pi(q)-1}\left\|\left.D f^{-1}\right|_{E^{u}\left(f^{-i}(q)\right)}\right\|<\lambda^{\pi(q)}
$$

(3) f has the shadowing property on $\mathrm{H}_{\mathrm{f}}(\mathrm{p})$.

Then $\mathrm{H}_{\mathrm{f}}(\mathrm{p})$ is hyperbolic.

End of the Proof of Theorem 1.2. Since $\mathrm{H}_{\mathrm{f}}(\mathrm{p})$ is R-robustly measure expansive, by Theorems 2.3 and 2.5, propositions (1) and (2) hold. By Proposition 3.5 and Bowen's result [3, Proposition 3.6], if the homoclinic class $H_{f}(p)$ is R-robustly measure expansive then $f$ has the shadowing property on $H_{f}(p)$, and so, proposition (3) also holds. Thus if $\mathrm{H}_{\mathrm{f}}(\mathrm{p})$ is R-robustly measure expansive then it is hyperbolic. 


\section{Acknowledgment}

This work is supported by Basic Science Research Program through the National Research Foundation of Korea (NRF) funded by the Ministry of Science, ICT \& Future Planning (No. 2017R1A2B4001892).

\section{References}

[1] A. Arbieto, Periodic orbits and expansiveness, Math. Z., 269 (2011), 801-807. 1

[2] C. Bonatti, S. Crovisier, Récurrence et généricité, Invent. Math., 158 (2004), 33-104. 2

[3] R. Bowen, Equilibrium States and the Ergodic Theory of Anosov Diffeomorphisms, Springer-Verlag, Berlin, (2008). 4

[4] M. W. Hirsch, C. C. Pugh, M. Shub, Invariant manifolds, Lecture Note in Math., Springer-Verlag, New York, (1977). 3.1

[5] H. Kato, Continuum-wise expansive homeomorphisms, Canad. J. Math., 45 (1993), 576-598. 1

[6] N. Koo, K. Lee, M. Lee, Generic diffeomorphisms with measure expansive homoclinic classes, J. Difference Equ. Appl., 20 (2014), 228-236. 1

[7] M. Lee, Measure expansiveness of the generic view point, preprint. 1

[8] K. Lee, M. Lee, Hyperbolicity of $\mathrm{C}^{1}$-stably expansive homoclinic classes, Discrete Contin. Dyn. Syst., 27 (2010), 11331145. 1

[9] K. Lee, M. Lee, Measure expansive homoclinic classes, Osaka J. Math., 53 (2016), 873-887. 1

[10] X. Li, On R-robustly entropy-expansive diffeomorphisms, Bull. Braz. Math. Soc., 43 (2012), 73-98. 1

[11] R. Mañẽ, Expansive diffeomorphisms, Lecture Notes in Math., Springer, Berlin, (1975). 1

[12] R. Mańẽ, Contribution to stability conjecture, Topology, 17 (1978), 383-396. 3.1

[13] C. Morales, A generalization of expansivity, Discrete Contin. Dyn. Syst., 32 (2012), 293-301. 1

[14] C. A. Morales, V. F. Sirvent, Expansive measures, Instituto Nacional de Matemtica Pura e Aplicada (IMPA), Rio de Janeiro, (2013). 1, 2

[15] M. J. Pacifico, E. R. Pujals, M. Sambarino, J. L. Vieites, Robustly expansive codimension-one homoclinic classes are hyperbolic, Ergodic Theory Dynam. Systems, 29 (2009), 179-200. 1

[16] M. J. Pacifico, E. R. Pujals, J. L. Vieites, Robustly expansive homoclinic classes, Ergodic Theory Dynam. Systems, 25 (2005), 271-300. 1

[17] M. J. Pacificao, J. L. Vieites, On measure expansive diffeomorphisms, Proc. Amer. Math. Soc., 143 (2015), 811-819. 1, 2

[18] K. Sakai, C ${ }^{1}$-stably shadowable chain components, Ergodic Theory Dynam. Systems, 28 (2008), 987-1029. 2

[19] K. Sakai, N. Sumi, K. Yamamoto, Measure expansive diffeomorphisms, J. Math. Anal. Appl., 414 (2014), 546-552. 1

[20] M. Sambarino, J. Vieitez, On $C^{1}$-persistently expansive homoclinic classes, Discrete Contin. Dynam. Syst., 14 (2006), 465-481. 1, 2, 2, 3

[21] M. Sambarino, J. Vieitez, Robustly expansive homoclinic classes are generically hyperbolic, Discrete Contin. Dynam. Syst., 24 (2009), 1325-1333. 3

[22] W. R. Utz, Unstable homeomorphisms, Proc. Amer. Math. Soc., 1 (1950), 769-774. 1

[23] X. Wen, S. Gan, L. Wen, $C^{1}$-stably shadowable chain components are hyperbolic, J. Differential Equations, 246 (2009), 340-357. 4.1 